\title{
Análisis de la comunicación en el paciente crónico hospitalizado y la mejora de su experiencia
}

\section{An analysis of the communication in hospitalized chronic patients and the improvement of their experience}

\author{
Guillem Marca-Francés; Joan Frigola-Reig; Marc Compte-Pujol
}

Cómo citar este artículo:

Marca-Francés, Guillem; Frigola-Reig, Joan; Compte-Pujol, Marc (2019). “Análisis de la comunicación en el paciente crónico hospitalizado y la mejora de su experiencia". El profesional de la información, v. 28, n. 2, e280221.

https://doi.org/10.3145/epi.2019.mar.21

Artículo recibido el 10-11-2018 Aceptación definitiva: 09-04-2019

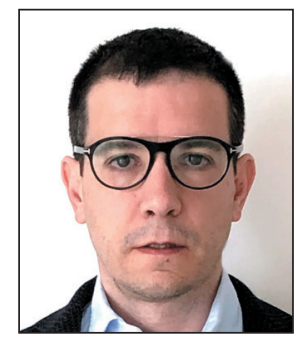

Guillem Marca-Francés $\bowtie$ https://orcid.org/0000-0002-1586-8625

Universitat de Vic-Universitat Central de Catalunya

Sagrada Família, 7. 08500 Vic

(Barcelona), España

guillem.marca@uvic.cat

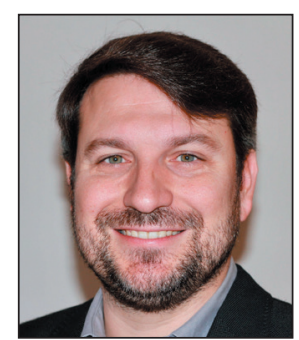

Joan Frigola-Reig

https://orcid.org/0000-0003-4195-1104

Universitat de Vic-Universitat Central de Catalunya

Sagrada Família, 7. 08500 Vic

(Barcelona), España

joan.frigola@uvic.cat

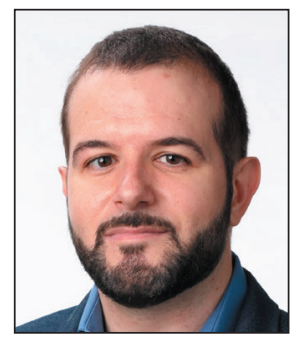

Marc Compte-Pujol

https://orcid.org/0000-0002-6694-2485

Universitat de Vic-Universitat Central de

Catalunya

Sagrada Família, 7. 08500 Vic

(Barcelona), España

marc.compte@uvic.cat

\section{Resumen}

Auditorías de hospitales estadounidenses incluyen la experiencia de paciente y la información entre proveedores de salud y pacientes. La comunicación efectiva promueve relaciones más sólidas entre pacientes y profesionales impactando en la calidad de la atención. Se analizaron los elementos y momentos más importantes de la comunicación de pacientes durante su hospitalización, como consecuencia de una reagudización de su patología crónica. Se realizaron grupos focales y entrevistas en profundidad con 30 pacientes ingresados de edades comprendidas entre 54 y 86 años. Los resultados identifican a médicos y enfermeras como los interlocutores más eficaces para la transmisión de la información, estableciendo diferencias. Los momentos de transmisión son la admisión, antes de las pruebas diagnósticas y en el momento del alta, y los temas de interés el diagnóstico y los síntomas. Los contenidos escritos permiten una mayor información comparado con la transmisión oral pues permiten múltiples lecturas.

Financiación

Este trabajo forma parte del proyecto de investigación Efectividad de una intervención de comunicación para la mejora de la experiencia del paciente crónico durante su estancia en el hospital (CSO2015-71419-R), proyecto competitivo de I+D financiado por el Ministerio de Ciencia, Innovación y Universidades de España. 


\title{
Palabras clave
}

Información de salud; Información médica; Comunicación de salud; Relación médico-paciente; Transmisión de información; Experiencia de paciente; Percepción de los pacientes; Hospitales; Enfermedades crónicas; Entrevistas; Grupos focales; Alfabetización en salud.

\begin{abstract}
American hospital audits include patient experience and information between health providers and patients. An effective communication promotes stronger relationships between patients and professionals, impacting on the quality of care. We analysed the most important communication elements and moments for patients during their hospitalization, as a consequence of an exacerbation of their chronic disease. Focus groups and in-depth interviews were conducted to 30 admitted patients between 54 and 86 years old. The results identify doctors and nurses as the most effective interlocutors for the transmission of information, establishing differences. Important moments were the admission, before the diagnostic tests and discharge, and the topics of interest are diagnosis and symptoms. Written contents enable to get more information compared to oral transmission because they allow multiple readings.
\end{abstract}

\section{Keywords}

Health information; Medical information; Health communication; Relationship doctor-patient; Information transmission; Patient experience; Patient perception; Hospitals; Chronic diseases; Interviews; Focus groups; Health literacy; Healthcare.

\section{Introducción}

Los hospitales gestionan continuamente cambios, que vienen dados tanto por cuestiones vinculadas a la evolución científico-clínica como por la aplicación de nuevas normativas o expectativas sociales. En 2009 la Organización Mundial de la Salud introdujo el concepto de experiencia de paciente, definido por The Beryl Institute como:

"la suma de todas las interacciones, moldeada por la cultura de una organización, que influyen en las percepciones del paciente a través de la continuidad de la atención" (Wolf et al., 2014).

La experiencia de paciente comenzó a conquistar el centro de la atención sanitaria estadounidense en 2014 (Salomon, 2014) y hoy la mayoría de evaluaciones de sus hospitales ya la incluyen, desarrollando una sección sobre la transmisión de la información entre proveedores de salud y pacientes (Lichstein, 2018).

Investigaciones previas han demostrado que la mayoría de la información médica presentada a los pacientes durante las visitas al hospital es deficiente (Kessels, 2003). Las limitaciones se deben a la propia información clínica, como la terminología o el formato (escrito/verbal) o al paciente, como su nivel educativo o unas expectativas específicas inadecuadas (Wilcox et al., 2010).

La capacidad de los pacientes para comunicarse efectivamente con el personal hospitalario es un elemento esencial del cuidado personal adecuado, especialmente para aquellos afectados por enfermedades crónicas (Clayman et al., 2010).

Los colectivos de personas mayores son más vulnerables en la obtención y el uso de información de salud; específicamente se asocian a niveles más bajos de educación, con mayor prevalencia de problemas de salud y un menor interés y proactividad en la búsqueda de información. Para las personas que cumplan con algunas de estas características es importante que la información disponible sobre salud sea comprensible y se pueda acceder a ella sin demasiado esfuerzo, algo que los interlocutores en los hospitales deben tener en cuenta (Eriksson-Backa et al., 2012). Los adultos mayores quieren ser escuchados y que sus preferencias se tengan en consideración (Williams-Roberts; Abonyi; Kryzanowski, 2018).

Por su parte, la comunicación efectiva promueve oportunidades para establecer relaciones más sólidas entre los pacientes, las familias y los médicos con el objetivo de brindar atención de alta calidad. La comunicación en la atención médica es fundamental para la experiencia del paciente y muestra el impulso institucional para mejorar la comunicación como un pilar fundamental para el éxito sistémico (Lichstein, 2018).

El principal objetivo de este trabajo es conocer cuáles son los elementos y los momentos más importantes para los pacientes durante su estancia en el hospital, como consecuencia de una reagudización de una patología crónica basal, abordables desde el ámbito de la comunicación. La intención es vincular aspectos de comunicación a una mejora de la experiencia del paciente, consiguiendo que la información entre profesionales asistenciales y pacientes no sea únicamente transmitida sino comprendida y retenida, es decir, puesta en valor para modificar actitudes que supongan mejoras en la adherencia al tratamiento y estilos de vida saludables. 


\section{Metodología}

Este artículo es la primera fase de un estudio realizado en el Consorcio Hospitalario de Vic, que se enmarca en un trabajo más amplio sobre la mejora de la experiencia en comunicación del paciente durante su estancia en el hospital.

El Consorcio Hospitalario de Vic es el hospital de referencia para 157.000 habitantes de la zona rural central de Catalunya, con unos 5.000 pacientes ingresados cada año.

Se plantearon cuatro preguntas de investigación:

PI1: ¿Cuáles son los interlocutores válidos para pacientes o cuidadores?

PI2: ¿Cuáles son los momentos clave para recibir eficazmente la información?

PI3: ¿Cuáles son los temas de interés para pacientes y cuidadores?

PI4: ¿Cuáles son los canales más adecuados para transmitir la información?

La PI1 pretendía conocer los interlocutores de pacientes y cuidadores en el hospital e identificar el rol que ejercen en el proceso de transmisión de la información.

La PI2 trataba de establecer en qué momentos había sido transmitida información por parte del hospital a pacientes o cuidadores, y cuál fue el nivel de efectividad de ésta.

La PI3 trató de identificar las temáticas transmitidas por el hospital y el nivel de interés que éstas suscitaban en pacientes y cuidadores. También se buscaron aquellos temas no tratados pero de interés para los pacientes.

La PI4 buscó establecer cuáles fueron los canales más adecuados para la comprensión y recuerdo de la información.

Para dar respuesta a las preguntas de investigación se seleccionaron dos metodologías cualitativas: el grupo focal y las entrevistas en profundidad.

Con el objetivo de garantizar unas características similares en el perfil de los participantes, se seleccionaron los que fueron ingresados después de una exacerbación de su condición crónica de EPOC (enterprise point-of-care) o insuficiencia cardíaca crónica. Se excluyeron los pacientes que padecían una enfermedad psico-mental que dificultara la comprensión de la información, y los que se encontraban en una etapa muy avanzada de su patología y estaban recibiendo cuidados paliativos.

Se realizaron dos grupos focales con 13 participantes en total (8 pacientes y 5 cuidadores). Los participantes habían sido dados de alta en las dos semanas previas a la actividad. Se realizaron después 17 entrevistas a pacientes mientras estaban ingresados.

A todos los participantes se les solicitó su participación en el estudio explicándoles los propósitos y el contenido del mismo. Después se les hizo firmar un consentimiento informado, antes de realizar la reunión o la entrevista. Todas las intervenciones se realizaron entre abril y junio de 2018.

Los datos aportados por los participantes fueron registrados y transcritos literalmente. Las sesiones duraron unos 90 minutos y las entrevistas 45.

\subsection{Análisis de datos}

Los pasos que se siguieron fueron los del análisis de contenido inductivo convencional. Las transcripciones verificadas fueron introducidas en el software Atlas.ti v7.5.13. Se realizó un primer análisis para tener una idea general de los contenidos y la relevancia de los temas planteados, así como de los enfoques considerados para el estudio. Después se segmentaron los datos organizando fragmentos que transmitieran una misma idea en unidades de significado. Dos investigadores leyeron de forma independiente las transcripciones buscando unidades de significado para permitir la indexación de segmentos que describían ideas similares. Éstos se compararon hasta llegar a un consenso y se procedió a la codificación de todas las transcripciones. Los datos obtenidos se organizaron en dos niveles:

- Nivel A: segmentación e identificación de unidades de significado, en categorías descriptivas;

- Nivel B: construcción de un sistema de temas, incluyendo varias unidades de significado o categorías.

\section{Resultados}

Se realizaron dos grupos focales de 6 y 7 participantes, de los cuales cuatro pacientes vinieron con un familiar (dos en el grupo 1 y dos en el grupo 2). También se realizaron 17 entrevistas en profundidad. Los participantes tenían edades comprendidas entre 54 y $86(\mu=73,5 ; S D=7,8)$. Todos los encuestados eran caucásicos y el $56,7 \%$ eran hombres ( $n=17)$.

El análisis cualitativo de la información obtenida en los grupos focales y las entrevistas proporcionaron 377 unidades de significado sintetizadas en 22 categorías y seis temas, siendo cuatro de ellos de interés para este trabajo, vinculados a las preguntas de investigación:

- interlocutores de los pacientes en el hospital;

- momentos clave; 
- niveles de conocimiento de su enfermedad (temas de interés);

- paquetes de información.

Los dos temas no desarrollados en este artículo fueron satisfacción del paciente y proactividad. El género del participante $(\mathrm{M} / \mathrm{F})$, la edad y el número de identificación del estudio se proporcionan entre paréntesis con las citas.

\subsection{Interlocutores con los pacientes}

Tres categorías identificaban a los interlocutores con los que los participantes de este estudio interactuaron. La categoría que más apareció fue personal de enfermería, en segundo lugar los profesionales médicos y por último "otros", que reunía personal administrativo y celadores (tabla 1 ).

Los participantes manifestaron de forma consensuada que el mayor número de contactos fue con el personal de enfermería (sin distinción entre enfermeras y auxiliares de enfermería), y que el contacto con el personal médico era mucho más puntual.

La relación de los pacientes con el personal de enfermería era constante en todos los momentos del paso por el hospital. Además este personal se en-

Tabla 1. Interlocutores de los pacientes

\begin{tabular}{|l|c|c|c|}
\cline { 2 - 4 } \multicolumn{1}{c|}{} & Entrevistas & Grupos focales & Total \\
\hline Interlocutores & 201 & 84 & 285 \\
\hline Médicos & 86 & 33 & 119 \\
\hline Enfermeras & 90 & 37 & 127 \\
\hline Otros & 25 & 14 & 39 \\
\hline
\end{tabular}
cargaba de humanizar la institución y los participantes lo recuerdan como el lado amable del hospital. Sin embargo también lo recuerdan esquivo en cuanto a la información que es clave para pacientes y cuidadores (enfermedad, manejo, tratamiento, etc.), como vemos en las siguientes declaraciones:

“Las enfermeras no me dieron información, no hablaron sobre la enfermedad, pero me animaron" [M, 77, 2]

“Cuando pregunté sobre los resultados de una prueba, la enfermera me dijo que el médico me hablaría” [M, 66, 10].

Estas respuestas del personal de enfermería refuerzan el valor de los médicos como interlocutores clave para la transmisión de información importante, aunque los pacientes recurran al personal de enfermería de forma repetida dado su fácil acceso.

Ante la pregunta de quién les transmitió la información importante, los pacientes reconocen la interlocución del facultativo:

"La otra vez estuve aquí (...) vino una doctora, bueno dos..." [M, 81, 13];

“El doctor me ha preguntado ‘¿se quiere ir?’ y le he respondido 'usted es la técnica y es usted quien lo puede decir', le he dicho 'yo me encuentro bien, no le engaño, si cree que me puedo ir, me iré', y me fui" [M, 66, 10].

No obstante, aun reconociendo la autoridad científica del médico, no tienen ningún problema con expresar su opinión y proponer discusiones sobre los temas de interés:

"Si viene la doctora mañana y me dice 'mañana le damos el alta', le diré que por mi parte no es necesario correr; prefiero salir de aquí completamente limpio" [M, 80, 8].

\subsection{Momentos clave}

Tanto en los grupos focales como en las entrevistas se mostró un amplio consenso sobre los momentos clave de la transmisión de información. Éstos fueron, por orden de menciones: el momento del alta hospitalaria, los procedimientos diagnósticos o terapéuticos durante la hospitalización, la estancia en urgencias y la estancia hospitalaria (tabla 2).

El momento del alta del hospital fue el elemento informativo con mayor presencia en las transcripciones (54 casos). Se puede aducir interés por este momento ya que la persona que transmite la información es el doctor, y detrás está el deseo de conocer cuándo será posible salir del hospital una Tabla 2. Momentos clave del proceso hospitalario

\begin{tabular}{|l|c|c|c|}
\cline { 2 - 4 } \multicolumn{1}{c|}{} & Entrevistas & Grupos focales & Total \\
\hline Momentos clave & 102 & 35 & 137 \\
\hline Estancia en urgencias & 22 & 7 & 29 \\
\hline Estancia hospitalaria & 8 & 4 & 12 \\
\hline Alta & 46 & 8 & 54 \\
\hline Procedimientos durante la hospitalización & 26 & 16 & 42 \\
\hline
\end{tabular}
vez se lleva unos días ingresado.

Si por un lado la presencia del momento del alta en las transcripciones es muy elevado, los recuerdos sobre el contenido informativo son generalmente parcos. Ante la insistencia del entrevistador sobre la información recibida en el alta, la respuesta de la mayoría de pacientes es: "es correcta, sí" [F, 65, 22].

La condición de pacientes crónicos de los participantes del estudio hace asumir que su entrada en el hospital es debida a un empeoramiento de su estado implicando una entrada en la mayoría de los casos por urgencias. Los participantes destacaron dos situaciones opuestas: 
1) la confianza de que en el hospital ya saben lo que tienen: “(...) ya saben lo que tengo, ya tienen el historial” [M, 81, 13]; 2) la falta de información sobre lo que está pasando: “Todo iba muy lento, no me daban información” [M, 72, 15].

La estancia hospitalaria en sí misma tuvo una presencia muy discreta en las transcripciones, presentándose únicamente en 12 casos. Los participantes las asocian al silencio informativo y a la falta de acción. Este silencio deriva en preguntas al personal de enfermería, como ya ha sido comentado en el punto anterior. Ante las preguntas de los entrevistadores sobre cuántos días más creen que estarán en el hospital, la respuesta mayoritaria, a no ser que se les haya comunicado el alta, es "no lo sé". Si han preguntado de forma insistente, han recibido respuestas no siempre alentadoras:

"Primero me dijeron unas horas, después veinticuatro, después te irás mañana, y al final llevo una semana" [M, $65,1]$.

Los participantes coinciden en que el personal hospitalario activa la información en los momentos de hacer pruebas diagnósticas, y algunos pacientes agradecen la paciencia, reconociendo su desconocimiento:

"A mí me lo han explicado todo bien, pero si una cosa no la he entendido, pregunto las veces que sean necesarias; a veces por lo que sea no lo entiendes bien y prefiero preguntar" [F, 70, 18],

mientras otros prefieren no preguntar por vergüenza. También transmiten temor por los resultados:

"Te hacen pruebas, y entonces... pueden salir muchas cosas de las pruebas" [M, 80, 3].

\begin{tabular}{|c|c|c|c|}
\hline & Médicos & Enfermeras & Otros \\
\hline Estancia en urgencias & 12 & 6 & 11 \\
\hline Estancia hospitalaria & 2 & 10 & 0 \\
\hline Alta & 23 & 15 & 14 \\
\hline $\begin{array}{l}\text { Procedimientos durante } \\
\text { la hospitalización }\end{array}$ & 18 & 19 & 5 \\
\hline
\end{tabular}

Figura 1. Relación de frecuencias entre momentos clave e interlocutores

En la figura 1 se puede observar la relación de frecuencias del estudio entre los interlocutores y los momentos clave. Destaca la hegemonía de la figura del médico en el proceso del alta y también en urgencias, cediendo el papel protagonista a enfermería en la estancia hospitalaria y los procedimientos y pruebas durante la hospitalización.

\subsection{Niveles de conocimiento}

Para abordar los temas de interés para los pacientes se optó por analizar cuáles recordaban y mencionaban sobre sus experiencias en su paso por el hospital. Las categorías más mencionadas fueron:

- el diagnóstico (52 casos);

- los antecedentes (43);

- las muestras de desconocimiento (40).

Las dos categorías más mencionadas, diagnóstico y antecedentes, están fuertemente relacionadas entre sí. Cuando se emplazó a los participantes a hablar de su situación, todos sin excepción recurrieron a presentar sus antecedentes, refiriéndose a diagnósticos que habían tenido en su historial pasado y obviando el momento presente:

"Comencé a cansarme y me dijeron que tenía que dejar de fumar" [F, 54, 7].

Tabla 3. Niveles de conocimiento del paciente sobre su enfermedad

\begin{tabular}{|l|c|c|c|}
\cline { 2 - 4 } \multicolumn{1}{c|}{} & Entrevistas & Grupos focales & Total \\
\hline Nivel de conocimiento del paciente sobre su enfermedad & 118 & 51 & 169 \\
\hline Antecedentes & 23 & 20 & 43 \\
\hline Diagnóstico & 38 & 14 & 52 \\
\hline Mejora de los síntomas & 13 & 4 & 17 \\
\hline Conocimiento sobre los síntomas & 13 & 4 & 17 \\
\hline Desconocimiento & 31 & 9 & 40 \\
\hline
\end{tabular}


Asimismo los pacientes asocian el diagnóstico con las primeras hospitalizaciones.

Las transcripciones muestran una mezcla de información entre síntomas, diagnósticos y saber popular:

“Me ardían las manos y la cabeza, me sentí extraño y pensé: si tengo fiebre, tengo una infección” [M, 83, 11].

Preguntados específicamente sobre el nombre de la enfermedad o los resultados de las pruebas que se realizaron, no suelen nombrar ninguna enfermedad específica y tampoco describir los resultados de las pruebas:

"No mencionaron una enfermedad que era mala y todo eso... nunca me han dicho que tengo algo, pero me dicen que tengo presión arterial alta, sangre alta, me enfrío casi todos los años... en los últimos tres años he estado en el hospital tres veces por lo mismo" [F, 86, 6].

Los participantes reconocen el desconocimiento y la falta de información en algunos casos:

“No me dieron información... me explicaron que estoy lo suficientemente bien para irme y que mi corazón está bien" [M, 80,8].

No obstante no mencionaron no poder entender al médico o enfermera, sino que construyeron relatos que argumentaban sus condiciones, patología y motivos de ingreso.

\begin{tabular}{|l|c|c|c|}
\cline { 2 - 4 } \multicolumn{1}{c|}{} & Médicos & Enfermeras & Otros \\
\hline Antecedentes & $\mathbf{2 4}$ & 16 & 3 \\
\hline Diagnóstico & $\mathbf{2 9}$ & 21 & 1 \\
\hline Mejora de los síntomas & 5 & 12 & 0 \\
\hline Conocimiento sobre los síntomas & 6 & $\mathbf{1 1}$ & 0 \\
\hline Desconocimiento & 6 & 19 & 5 \\
\hline
\end{tabular}

Figura 2. Relación de frecuencias entre niveles de conocimiento del paciente sobre su enfermedad e interlocutores

A través de los contenidos de sus niveles de conocimiento se identificaron los temas de interés como el diagnóstico, los síntomas y la evolución de la enfermedad. Con los contenidos del código de desconocimiento, se identificaron deficiencias informativas vinculadas a las patologías que padecían los pacientes y a los equipos que los asistían.

Contextualizando las categorías de las conversaciones mantenidas con los interlocutores antes o después de la mención, podemos concluir que las categorías de antecedentes y diagnóstico están fuertemente vinculadas con el profesional médico, y la categoría mejora de síntomas, conocimiento de síntomas o desconocimiento a enfermería (figura 2). Esto se debe sobre todo a que las informaciones de antecedentes de la primera patología y los datos de diagnóstico se recuerdan de las interlocuciones con los médicos. Enfermería por su parte pregunta sobre el estado del paciente y éste asocia la respuesta que él mismo da o las preguntas que realiza a los distintos interlocutores.

\subsection{Paquetes de información}

Este tema agrupó los códigos (tabla 4):

- comprensión de la información (74 casos);

- información aportada por el médico (69);

- información en la admisión (48);

- falta de información (13).

En la mayoría de los casos la inforTabla 4. Paquetes de información

\begin{tabular}{|l|c|c|c|}
\cline { 2 - 4 } \multicolumn{1}{c|}{} & Entrevistas & Grupos focales & Total \\
\hline Paquetes de información & 163 & 41 & 204 \\
\hline Información en la admisión & 37 & 11 & 48 \\
\hline Información aportada por el médico & 53 & 16 & 69 \\
\hline Comprensión de la información & 63 & 11 & 74 \\
\hline Falta de información & 10 & 3 & 13 \\
\hline
\end{tabular}
mación aportada por los médicos es indiscutible, la asumen como una verdad absoluta de buen grado, sin necesidad de comprender exactamente lo que realmente tienen o les hacen:

"El médico te dice: hoy no te vas, pensaba que te irías hoy... te irás el lunes porque te veo muy flaco y te falta esto o te falta aquello; no sé qué decir exactamente" [M, 77, 17], 
"Me han dejado unos papeles, me han dado el alta... no lo he acabado de entender. Pero bueno, es igual. Supongo que ya estoy bien" [M, 65, 1],

"El médico ya tiene los resultados de todo, me ha encontrado una cosa que parece que tengo aquí, pero que no tiene importancia. Me lo he de seguir mirando. Tengo un pequeño bulto pero estoy bien" [M, 83, 11].

Además algunos participantes admiten que la información escrita pueden digerirla y comprenderla con el tiempo, cuando están solos, comentándola con familiares pero no ante el médico:

"Las pautas son muy claras. Nunca he tenido problemas en entenderlas. Recuerdo una vez que me hicieron un cuadro muy chulo, se veía muy bien con las pastillas de cada hora" [M, 74, 5].

Aunque después actúan con total libertad:

"Cuando me voy a casa no sigo las pautas a pies juntillas, las que me dieron eran muy parecidas a las que ya tenía, sigo con aquéllas" [M, 74, 5].

Cuando se interpeló a los pacientes sobre la información recibida previa al ingreso, en la admisión en el hospital, todos admitieron que fueron informados correctamente de lo que tenían, no obstante los recuerdos fueron vagos y confusos. Debemos recordar que este ingreso se produjo en todos los casos por urgencias debido a un empeoramiento de sus patologías crónicas.

La falta de información se presenta en dos formatos muy diferenciados. Por un lado, se obvia por parte de los clínicos la necesidad de explicaciones a los pacientes y éstos no la piden, ya sea por vergüenza o inseguridad:

“El médico no me dijo nunca nada, solamente me preguntó si estaba bien, me miró la fiebre... 'Quizá esta tarde sales'. Fue a buscar a otro médico más joven, se lo miraron un rato, hablaron entre ellos con papeles, y ya está" $[\mathrm{M}, 80,16]$.

Pasan situaciones similares en las que los pacientes se identifican con mensajeros:

"El doctor me dijo 'toma estos papeles para el médico de cabecera, pasas por allá en una semana y se los puedes dar... bueno, ellos ya lo tienen en el ordenador también'" [M, 83, 11].

Por otro lado, algunos pacientes admiten que las faltas de información las suplen consultando (o pidiendo a familiares que consulten) internet y las redes sociales, aunque transmitiendo prudencia:

"Busqué vía internet y vía todo... yo que sé, todo lo que se puede encontrar en internet, tampoco se puede hacer mucho caso" [M, 83, 9].

Otros afirman que ante las dudas prefieren preguntar al facultativo.

La mayoría de la información fue transmitida de forma oral, tanto la aportada en el momento de la admisión como la transmitida por los clínicos, exceptuando los informes o resúmenes de pautas entregados en el momento previo al alta. La mayoría de participantes valoraron positivamente la información escrita.

\begin{tabular}{|l|c|c|c|}
\cline { 2 - 4 } \multicolumn{1}{c|}{} & Médicos & Enfermeras & Otros \\
\hline Información en la admisión & 13 & 7 & 5 \\
\hline Información aportada por el médico & 69 & 0 & 0 \\
\hline Comprensión de la información & 39 & 26 & 0 \\
\hline Falta de información & 10 & 1 & 0 \\
\hline
\end{tabular}

Figura 3. Relación de frecuencias entre paquetes de información e interlocutores

Si relacionamos los paquetes de información con los interlocutores, los pacientes establecen un vínculo directo con el profesional médico tanto en las cuestiones positivas (comprensión de la información) como en las negativas, muchas menos (falta de información). Los interlocutores pasan totalmente desapercibidos en el contaje de frecuencias (figura 3). 


\begin{tabular}{|l|c|c|}
\cline { 2 - 3 } \multicolumn{1}{c|}{} & Comprensión de la información & Falta de información \\
\hline Estancia en urgencias & 19 & 3 \\
\hline Estancia hospitalaria & 6 & 4 \\
\hline Alta & 29 & 3 \\
\hline $\begin{array}{l}\text { Procedimientos durante } \\
\text { la hospitalización }\end{array}$ & 20 & 3 \\
\hline
\end{tabular}

Figura 4. Relación de frecuencias entre momentos clave y comprensión o falta de información

Por otra parte, la relación de las frecuencias entre momentos clave y paquetes de información, y la relación entre estancia en urgencias y la información de la admisión, coinciden en su práctica totalidad. También coinciden la información aportada por el médico con el mismo análisis realizado más arriba entre momentos clave e interlocutores en la columna médicos. Los nuevos datos que nos aporta esta relación son que los momentos relacionados con la comprensión de la información son el alta, los procedimientos durante la hospitalización y la estancia en urgencias, dejando en una frecuencia muy baja la estancia en el hospital. Con frecuencias mucho más inferiores, los momentos se correlacionan de la misma forma con la falta de información (figura 4).

\section{Discusión y conclusiones}

Se observa que los pacientes en el circuito hospitalario identifican dos únicos interlocutores clave: facultativos y enfermeros. Ambos perfiles son proveedores de información y ampliamente respetados, pero en algunas ocasiones el recuerdo de médicos y enfermeras queda sin llegar a asociar sus nombres. Esta situación se planteó de una forma más clara con enfermería.

Médicos y enfermeros se complementan, aportando el primer colectivo informaciones mucho más racionales y de carácter vital para el paciente, mientras que enfermería aporta un aspecto más afectivo. Esta situación no debería darse de esta forma, y ambos perfiles deberían ser percibidos con la misma carga de afecto, pues la comunicación afectiva se relaciona positivamente con las competencias de transmisión de información de los proveedores de salud y la adhesión al tratamiento de los pacientes mientras que la privación de afecto tiene resultados opuestos (Hess; Rauscher, 2018).

Los resultados también muestran que el colectivo "otros" (personal de administración, limpieza, celadores, camilleros, etc.) aparece muy poco. Este dato contradice los planteamientos de Kennedy, Didehban y Fasolino (2014) que afirman que dejar de lado al resto de interlocutores presentes en el hospital, contradice las tendencias del movimiento de experiencia de paciente en el que todo lo que lo rodea interviene en su experiencia. Cabe una reflexión al respecto del motivo por el que no aparecen y las políticas de interlocución y relaciones entre el personal y los pacientes y cuidadores a desarrollar en los centros hospitalarios.

Los momentos identificados en los códigos de nuestro trabajo coinciden en parte con el proceso de ingreso hospitalario, con pequeños matices, a la vez que aportan información valiosa referente a los momentos que más se repiten: el alta y los procesos internos. Para los pacientes el momento del alta es importante desde el punto de vista informativo; desean saber cómo salen del hospital en términos de evolución de su patología, qué pautas deben seguir y si hay tratamiento asociado al alta. Este momento también es importante desde el punto de vista clínico, ya que supone un punto de inflexión en la transmisión de información sobre el paciente, no resuelto con un éxito muy comedido en España (Marca-Francés et al., 2014). Algunos estudios abordan este momento como un desafío, ya que debe producirse información en procesos paralelos y adaptable a diferentes destinatarios: el paciente y el sistema (otros clínicos). Además, proporcionar a los pacientes únicamente información que se adapte a ellos descuida el interés que éstos pueden tener en ver qué información sobre ellos se transmite a la atención primaria (Wibe; Ekstedt; Helles $\varnothing$, 2015). Ha quedado expuesto por alguno de los pacientes su papel de mensajeros entre ambos estamentos del sistema, el hospital y el centro de atención primaria, sin identificar este hecho como negativo, simplemente se asume.

La estancia en urgencias se presentó en pocos casos con desconocimiento o falta de información. Björvell y Stieg (1991) demostraron que los pacientes que recibieron más información durante su estancia en urgencias, en particular información sobre qué esperar a continuación, calificaron su experiencia hospitalaria significativamente más positiva que 
aquellos que no tuvieron acceso a esta información mientras recibían atención. Los pacientes tienden a estar tan preocupados por los aspectos psicológicos y sociales de su estado como por los factores físicos. Desafortunadamente se ha demostrado que esta ansiedad está correlacionada con resultados clínicos negativos, tanto en el tratamiento crónico como en el de emergencia (Wilcox et al., 2010). Por estos motivos, aunque las frecuencias encontradas en la estancia en urgencias son bajas, es importante establecer protocolos claros de transmisión informativa, incluso formativa, pues es el momento clave en el que mucha de la información se presenta por primera vez a los pacientes y cuidadores.

Los resultados también muestran frecuencias muy bajas en la estancia hospitalaria. Además, los datos de comprensión de la información o falta de información son ínfimos. En principio y de la forma que han sido clasificadas las categorías de momentos clave, la estancia hospitalaria no tiene contenido en sí misma, pues las informaciones se transmiten al llegar y al salir de los centros hospitalarios o en las pruebas diagnósticas o intervenciones. En la estancia, pacientes y familiares únicamente consumen información sobre la evolución de su estado, y la información normalmente es poca. Estos momentos de estancia en planta, en los que el flujo informativo es vago, se puede identificar como una oportunidad.

De cara a posibles intervenciones, las estancias en planta pueden ser rellenadas con píldoras informativas, referentes a los elementos sobre los que los pacientes se muestran más desinformados teniendo tiempo para la reflexión. Los datos aportados por algunos participantes en cuanto a los canales utilizados dejan entrever el uso predominante de la técnica oral en la recepción de la información, siendo el uso de material gráfico o escrito una excepción. En esta línea, el sentimiento de propiedad de aquello que el paciente se queda o puede ver reflexivamente, mejora sus perspectivas de comprensión. Los estudios han demostrado que la información escrita que documenta la consulta médica se recuerda mejor y es más probable que resulte en adhesión a la atención (Blinder et al., 2001). En el proceso hospitalario esto sucede únicamente en el momento del alta, en el que los pacientes no centran la atención en lo que se les comunica oralmente, pero se muestran tranquilos al contar con un informe entre sus manos.

El alta es el momento clave más destacado, con un protagonismo muy elevado de los facultativos y también el más destacado en cuanto a comprensión y/o falta de información. En el momento del alta los pacientes experimentan una transición abrupta desde la participación pasiva en el servicio de urgencias a la responsabilidad absoluta de su cuidado en el momento del alta (Bickmore; Pfeifer; Jack, 2009).

Después del alta, o incluso a veces en la estancia en el hospital, la insatisfacción sobre la información recibida, que depende la mayoría de las veces de la empatía mostrada por el facultativo y el tiempo que ha dedicado al paciente, puede llevar en algunos casos a la consulta de contenidos en internet, e incluso a confiar en estos últimos (Majerovitz et al., 1997). Los adultos mayores buscan más información de salud en internet que los adultos más jóvenes y están más inclinados a leer materiales para obtener una visión general de su salud, aunque no confían en comprender la mayor parte del contenido (Huvila et al., 2018).

La comunicación efectiva es una función esencial en las interacciones entre el paciente y el proveedor a lo largo de la atención continua (Williams-Roberts; Abonyi; Kryzanowski, 2018). Explorar opciones de transmisión informativa durante los momentos de la estancia en planta para facilitar contenidos (escritos o visuales) a los pacientes sobre su patología, podría suavizar la transición del hospital a casa. Esta contribución a la materialización del derecho a la información y a la formación en aspectos relacionados con la salud y la enfermedad, ya la propusieron Vall y Rodríguez-Parada (2010) desde el entorno de la documentación.

Las evidencias de nuestro estudio confirman que los interlocutores para la transmisión de la información a los pacientes son médicos y enfermeras, y que dicha información debería transmitirse en la admisión en el hospital (aunque sea en urgencias, y de la forma más rápida posible para evitar la ansiedad) antes de las pruebas diagnósticas y en el momento del alta. Los pacientes recuerdan sobre todo la información de su diagnóstico y los síntomas que han vivido, pero muestran desinformación sobre la patología que sufren y sobre el entorno en el que se encuentran. Proponemos utilizar los momentos de estancia en la habitación para dar una visión general de la patología a los pacientes, quizá de forma escrita, visual o audiovisual.

\section{Limitaciones del estudio}

Las limitaciones son diversas. Siendo 30 los pacientes con los que se contó, la dispersión entre 2 grupos focales y 17 entrevistas en profundidad ha hecho prevalecer el interés por ahondar en la temática tratada más que en la representatividad de la muestra. En la misma línea, el hecho de centrarse en la comunicación y la transmisión de información ha dejado de lado otros aspectos de la experiencia del paciente que podrían ser de interés.

Otro elemento a tener en cuenta en la tabulación e interpretación de los resultados más allá del uso de dos técnicas complementarias, es el marco temporal de las intervenciones en referencia a la estancia al hospital. Los miembros del grupo focal habían recibido el alta en los 15 días previos a la sesión, mientras que las entrevistas en profundidad se reali- 
zaron durante el ingreso. En un principio el perfil de los sujetos demandaba la recopilación de datos durante la estancia, para evitar el sesgo de recuerdo, pero el momento del alta podía no haber sido tratado en la mayoría de los casos y por ello se consensuó complementar ambas técnicas. Una vez realizada la recopilación de información, se pudo observar que el perfil crónico de los pacientes suponía reingresos, y por ello respondieron a cuestiones de experiencias pasadas.

La elevada edad de los participantes, su origen rural y la afectación por una patología crónica fue otra de las limitaciones. Todas estas características han dificultado la recopilación de la información debido a la necesidad constante de centrar los temas además de limitar los hallazgos de nuestro estudio precisamente a esta subpoblación. No obstante, debido a la cronicidad se trata de pacientes que conocen el sistema y que son consumidores de servicios sanitarios, convirtiéndoles en expertos a nivel de vivencias hospitalarias.

\section{Referencias}

Bickmore, Timothy W.; Pfeifer, Laura M.; Jack, Brian W. (2009). "Taking the time to care: empowering low health literacy hospital patients with virtual nurse agents". In: $\mathrm{CHI}$ '09 Proceedings of the SIGCHI Conference on human factors in computing systems, pp. 1265-1274.

https://doi.org/10.1145/1518701.1518891

Björvell, Hjordis; Stieg, J. (1991). "Patients' perceptions of the health care received in an emergency department". Annals of emergency medicine, v. 20, n. 7, pp. 734-738.

https://doi.org/10.1016/S0196-0644(05)80833-9

Blinder, Danielle; Rotenberg, Leif; Peleg, Michael; Taicher, Shlomo (2001). "Patient compliance to instructions after oral surgical procedures". International journal of oral and maxillofacial surgery, v. 30, n. 3, pp. 216-219.

https://doi.org/10.1054/ijom.2000.0045

Clayman, Marla L.; Pandit, Anjali U.; Bergeron, Ashley R.; Cameron, Kenzie A.; Ross, Emily; Wolf, Michael S. (2010). "Ask, understand, remember: A brief measure of patient communication self-efficacy within clinical encounters". Journal of health communication, v. 15, n. sup. 2, pp. 72-79.

https://doi.org/10.1080/10810730.2010.500349

Eriksson-Backa, Kristina; Ek, Stefan; Niemelä, Raimo; Huotari, Maija-Leena (2012). "Health information literacy in everyday life: A study of Finns aged 65-79 years". Health informatics journal, v. 18, n. 2, pp. 83-94.

https://doi.org/10.1207/1460458212445797

Hesse, Colin; Rauscher, Emily A. (2018). "The relationships between doctor-patient affectionate communication and patient perceptions and outcomes". Health communication.

https://doi.org/10.1080/10410236.2018.1439269

Huvila, Isto; Enwald, Heidi; Eriksson-Backa, Kristina; Hirvonen, Noora; Nguyen, Hay; Scandurra, Isabella (2018). “Anticipating ageing: Older adults reading their medical records". Information processing \& management, v. 54, n. 3, pp. 394-407.

https://doi.org/10.1016/j.ipm.2018.01.007

Kennedy, Denise M.; Didehban, Roshanak; Fasolino, John P. (2014). "Creating and sustaining a culture of accountability for patient experience". Patient experience journal, v. 1, n. 2, art 9.

https://pxjournal.org/journal/vol1/iss2/9

Kessels, Roy P. C. (2003). "Patients' memory for medical information". Journal of the Royal Society of Medicine, v. $96, \mathrm{n}$. 5, pp. 219-222.

https://doi.org/10.1177/014107680309600504

Lichstein, Peter R. (2018). "Communication and patient experience”. In: Chou, Calvin L.; Cooley, Laura. Communication Rx: Transforming healthcare through relationship-centered communication, pp. 13-25. ISBN: 9781260019759

Majerovitz, S. Deborah; Greene, Michele G.; Adelman, Ronald D.; Brody, Gerald M.; Leber, Kathleen; Healy, Susan W. (1997). "Older patients' understanding of medical information in the emergency department". Health communication, v. 9, n. 3, pp. 237-251.

https://doi.org/10.1207/s15327027hc0903_3

Marca-Francés, Guillem; Pérez, Ángel J.; Blanco-García, Martín-Germán; Miravalles, Elena; Soley, Pere; Ortiga, Berta (2014). "The use of electronic health records in Spanish hospitals". Health information management journal, v. 43, n. 3, pp. 37-44.

https://doi.org/10.1177/183335831404300305

Salomon, Micah (2014). "9 patient experience and satisfaction secrets for hospitals and healthcare". Forbes, 8 Sept. https://www.forbes.com/sites/micahsolomon/2014/09/08/7-things-hospitals-and-healthcare-professionals-get-wrongabout-the-patient-experience-and-patient-satisfaction 
Vall, Aurora; Rodríguez-Parada, Concepción (2010). “Información para pacientes y educación en la salud de la ciudadanía: a la búsqueda de nuevos usuarios". El profesional de la información, v. 19, n. 3, pp. 296-299.

https://doi.org/10.3145/epi.2010.may.11

Wibe, Torunn; Ekstedt, Mirjam; Helles $\varnothing$, Ragnhild (2015). “Information practices of health care professionals related to patient discharge from hospital". Informatics for health and social care, v. 40, n. 3, pp. 198-209.

https://doi.org/10.3109/17538157.2013.879150

Wilcox, Lauren; Morris, Dan; Tan, Desney; Gatewood, Justin (2010). “Designing patient-centric information displays for hospitals". In: Procs of the SIGCHI Conference on human factors in computing systems, Atlanta, pp. 2123-2132.

https://dl.acm.org/authorize.cfm?key=242215

Williams-Roberts, Hazel; Abonyi, Sylvia; Kryzanowski, Julie (2018). "What older adults want from their health care providers". Patient experience journal, v. 5, n. 3, pp. 81-90.

https://pxjournal.org/journal/vol5/iss3/11

Wolf, Jason A.; Niederhauser, Victoria; Marshburn, Dianne; LaVela, Sherri L. (2014). "Defining patient experience". Patient experience journal, v. 1, n. 1, pp. 6-19.

http://pxjournal.org/journal/vol1/iss1/3

\section{Colección de libros de bolsillo El profesional de la información (Editorial UOC) últimos títulos publicados}
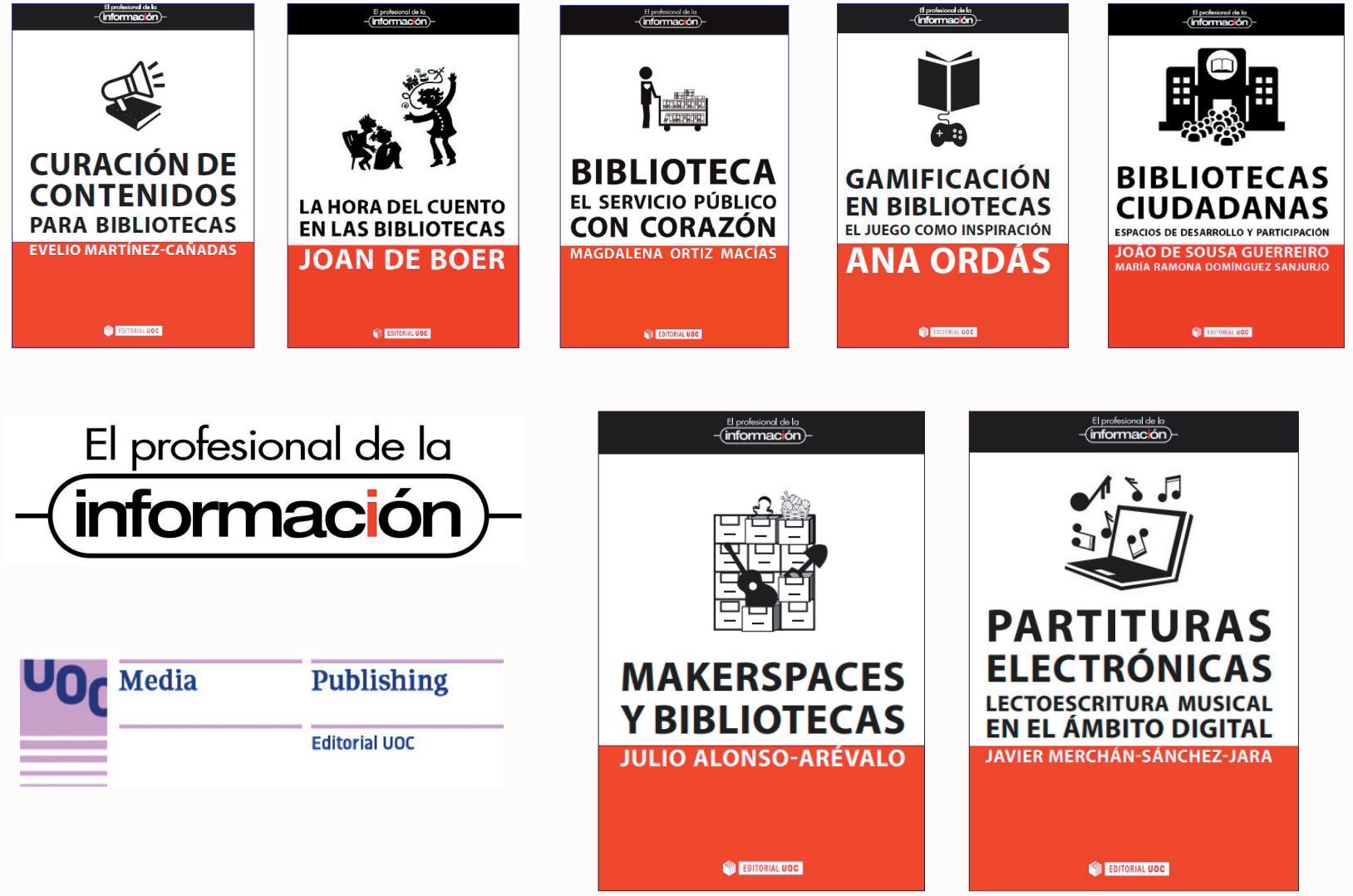

Más información:

http://www.elprofesionaldelainformacion.com/libros.html 


\section{$9-7,7 x$ धा}

$(-5)$ - 1 A

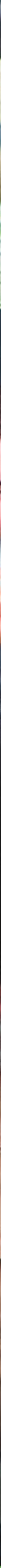

20 años diseñando

y gestionando información

MASmedios apoya la Declaracion de Lyon del 2014 que

propugna el derecho de las personas a acceder a la información.

\section{masmedios}

www.masmedios.com 